APS/123-QED

\title{
Statistical Mechanical Approach to Lossy Data Compression: Theory and Practice
}

\author{
Tadaaki Hosakd* \\ National Institute of Advanced Industrial Science and Technology \\ Tsukuba, Japan 3058568 \\ Yoshiyuki Kabashima \\ Department of Computational Intelligence and Systems Science \\ Tokyo Institute of Technology, Yokohama, Japan 2268502
}

(Dated: April 24, 2022)

\begin{abstract}
The encoder and decoder for lossy data compression of binary memoryless sources are developed on the basis of a specific-type nonmonotonic perceptron. Statistical mechanical analysis indicates that the potential ability of the perceptron-based code saturates the theoretically achievable limit in most cases although exactly performing the compression is computationally difficult. To resolve this difficulty, we provide a computationally tractable approximation algorithm using belief propagation (BP), which is a current standard algorithm of probabilistic inference. Introducing several approximations and heuristics, the BP-based algorithm exhibits performance that is close to the achievable limit in a practical time scale in optimal cases.
\end{abstract}

PACS numbers: $89.70 .+\mathrm{c}, 75.10 . \mathrm{Nr}, 02.50 . \mathrm{Tt}$

*Electronic address: hosaka-t@aist.go.jp 


\section{INTRODUCTION}

Lossy data compression is a core technology of contemporary broadband communication. The best achievable performance of lossy compression is theoretically provided by rate-distortion theorems, which were first proved by Shannon for memoryless sources [1]. Unfortunately, Shannon's proofs are not constructive and suggest few clues for how to design practical codes. Consequently, no practical schemes saturating the potentially optimal performance of lossy compression represented by the rate-distortion function (RDF) have been found yet, even for simple information sources. Therefore, the quest for better lossy compression codes remains a major problem in the field of information theory (IT).

Recent research on error correcting codes has revealed a similarity between IT and statistical mechanics (SM) of disordered systems [2]. Because it has been shown that methods from SM can be useful to analyse subjects of IT, it is natural to expect that a similar approach might also bring about novel developments in lossy compression.

This research is promoted by such a motivation. Specifically, we propose a simple compression code for uniformly biased binary data devised on input-output relations of a perceptron. Theoretical evaluation based on the replica method (RM) indicates that this code potentially saturates the RDF in most cases although exactly performing the compression is computationally difficult. To resolve this difficulty, we develop a computationally tractable algorithm based on belief propagation (BP) [3], which offers performance that approaches the RDF in a practical time scale when optimally tuned.

\section{LOSSY DATA COMPRESSION}

We describe a general scenario for lossy data compression of memoryless sources. Original data is denoted as $\boldsymbol{y}=\left(y^{1}, y^{2}, \ldots, y^{M}\right)$, which is assumed to comprise a sequence of $M$ discrete or continuous random variables that are generated independently from an identical stationary distribution $p(y)$. The purpose of lossy compression is to compress $\boldsymbol{y}$ into a binary expression $\boldsymbol{s}=\left(s_{1}, s_{2}, \ldots, s_{N}\right)\left(s_{i} \in\{+1,-1\}\right)$, allowing a certain amount of distortion between the original data $\boldsymbol{y}$ and its representative vector $\tilde{\boldsymbol{y}}=\left(\tilde{y}^{1}, \tilde{y}^{2}, \ldots, \tilde{y}^{M}\right)$ when $\tilde{\boldsymbol{y}}$ is retrieved from $s$.

In this study, distortion is measured using a distortion function that is assumed to be 
defined in a component-wise manner as $\mathcal{D}(\boldsymbol{y}, \tilde{\boldsymbol{y}})=\sum_{\mu=1}^{M} d\left(y^{\mu}, \tilde{y}^{\mu}\right)$, where $d\left(y^{\mu}, \tilde{y}^{\mu}\right) \geq 0$. A code $\mathcal{C}$ is specified by a map $\tilde{\boldsymbol{y}}(\boldsymbol{s} ; \mathcal{C}): \boldsymbol{s} \rightarrow \tilde{\boldsymbol{y}}$, which is used in the restoration phase. This map also reasonably determines the compression phase as

$$
\boldsymbol{s}(\boldsymbol{y} ; \mathcal{C})=\underset{\boldsymbol{s}}{\operatorname{argmin}}\{\mathcal{D}(\boldsymbol{y}, \tilde{\boldsymbol{y}}(\boldsymbol{s} ; \mathcal{C}))\}
$$

where $\operatorname{argmin}_{\boldsymbol{s}}\{\cdots\}$ represents the argument $s$ that minimises $\cdots$. When $\mathcal{C}$ is generated from a certain code ensemble, typical codes satisfy the fidelity criterion

$$
\frac{1}{M} \min _{\boldsymbol{s}}\left\{\mathcal{D}(\boldsymbol{y}, \tilde{\boldsymbol{y}}(\boldsymbol{s} ; \mathcal{C})\}=\frac{1}{M} \min _{\boldsymbol{s}}\left\{\sum_{\mu=1}^{M} d\left(y^{\mu}, \tilde{y}^{\mu}(\boldsymbol{s} ; \mathcal{C})\right)\right\}<D\right.
$$

for a given permissible distortion $D$ and typical original data $\boldsymbol{y}$ with probability 1 in the limit $M, N \rightarrow \infty$ maintaining the coding rate $R \equiv N / M$ constant, if and only if $R$ is larger than a certain critical rate $R_{c}(D)$ that is termed the rate-distortion function.

However, for finite $M$ and $N$, any code has a finite probability $P_{\mathrm{F}}$ of breaking the fidelity (2), even for $R>R_{c}(D)$. Similarly, for $R<R_{c}(D)$, Eq. (2) is satisfied with a certain probability $P_{\mathrm{S}}$. For reasonable code ensembles, the averages of these probabilities are expected to decay exponentially with respect to $M$ when the data length $M$ is sufficiently large. Therefore, the two error exponents $\alpha_{A}(D, R)=\lim _{M \rightarrow \infty}-(1 / M) \ln \left\langle P_{\mathrm{F}}\right\rangle_{\mathcal{C}}$ for $R>R_{c}(D)$ and $\alpha_{B}(D, R)=\lim _{M \rightarrow \infty}-(1 / M) \ln \left\langle P_{\mathrm{S}}\right\rangle_{\mathcal{C}}$ for $R<R_{c}(D)$, where $\langle\cdots\rangle_{\mathcal{C}}$ represents the average over the code ensemble, can be used to characterise the potential ability of the ensemble of finite data lengths.

\section{COMPRESSION BY PERCEPTRON AND THEORETICAL EVALUATION}

It is conjectured that the components of $s$ are preferably unbiased and uncorrelated in order to minimise loss of information in the original data from a binary information source, which implies that the entropy per bit in $s$ must be maximised. On the other hand, in order to reduce the distortion, the representative vector $\tilde{\boldsymbol{y}}(\boldsymbol{s} ; \mathcal{C})$ should be placed close to the typical sequences of the original data that are biased. Unfortunately, it is difficult to construct a code that satisfies these two requirements using only linear transformations over the Boolean field because a linear transformation generally reduces statistical bias in sequences, which implies that one cannot produce a biased representative vector from the unbiased compressed sequence. 
One method to design a code that has the above properties is to introduce a nonlinear transformation. A perceptron provides a simple scheme for carrying out this task. To specify lossy data compression codes for binary original data $\boldsymbol{y} \in\{+1,-1\}^{M}$ generated from a memoryless source, we define a map by utilising perceptrons from the compressed expression $\boldsymbol{s} \in\{+1,-1\}^{N}$ to the representative sequence $\tilde{\boldsymbol{y}}(\boldsymbol{s} ; \mathcal{C}) \in\{+1,-1\}^{M}$ as

$$
\tilde{y}^{\mu}\left(\boldsymbol{s} ;\left\{\boldsymbol{x}^{\mu}\right\}\right)=f\left(\frac{1}{\sqrt{N}} \sum_{i=1}^{N} x_{i}^{\mu} s_{i}\right), \quad(\mu=1,2, \ldots, M)
$$

where $f(\cdot)$ is a function for which the output is limited to $\{+1,-1\}$ and $\boldsymbol{x}^{\mu=1,2, \ldots, M}$ are randomly predetermined $N$-dimensional vectors that are generated from an $N$-dimensional normal distribution $P(\boldsymbol{x})=(\sqrt{2 \pi})^{-N} \exp \left[-|\boldsymbol{x}|^{2} / 2\right]$. These vectors are known to the encoder and decoder. We adopt an output function $f_{k}(u)=1$ for $|u|<k$, and -1 otherwise, which eventually offers optimal performance.

We measure the distortion by the Hamming distance $\mathcal{D}\left(\boldsymbol{y}, \tilde{\boldsymbol{y}}\left(\boldsymbol{s} ;\left\{\boldsymbol{x}^{\mu}\right\}\right)\right)=$ $\sum_{\mu=1}^{M}\left[\left\{1-y^{\mu} \cdot f_{k}\left(\sum_{i=1}^{N} x_{i}^{\mu} s_{i} / \sqrt{N}\right)\right\} / 2\right]$. Then, the compression phase for the given data $\boldsymbol{y}$ can be defined as finding a vector $s$ that minimises the resulting distortion $\mathcal{D}\left(\boldsymbol{y}, \tilde{\boldsymbol{y}}\left(\boldsymbol{s} ;\left\{\boldsymbol{x}^{\mu}\right\}\right)\right)$, and the retrieval process can be performed easily using Eq. (3) from a given sequence $\boldsymbol{s}$. The performance evaluation has been investigated theoretically from the perspective of SM, which is not specialised for this perceptron-based code. Rather, it is a general one as mentioned briefly below.

Let us regard the distortion function $\mathcal{D}(\boldsymbol{y}, \tilde{\boldsymbol{y}}(\boldsymbol{s} ; \mathcal{C}))$ as the Hamiltonian for the dynamical variable $\boldsymbol{s}$, which also depends on predetermined variables $\boldsymbol{y}$ and $\mathcal{C}$. The resulting distortion (per bit) for a given $\boldsymbol{y}$ and $\mathcal{C}$ is represented as $\lambda(\boldsymbol{y}, \mathcal{C})=\min \boldsymbol{s}\left\{M^{-1} \mathcal{D}(\boldsymbol{y}, \tilde{\boldsymbol{y}}(\boldsymbol{s} ; \mathcal{C}))\right\}$. We start with a statistical mechanical inequality

$$
e^{-M \beta \lambda(\boldsymbol{y}, \mathcal{C})} \leq \sum_{\boldsymbol{s}} e^{-\beta \mathcal{D}(\boldsymbol{y}, \tilde{\boldsymbol{y}}(\boldsymbol{s} ; \mathcal{C}))}=Z(\beta ; \boldsymbol{y}, \mathcal{C})=e^{-M \beta f(\beta ; \boldsymbol{y}, \mathcal{C})},
$$

which holds for any sets of $\beta>0, \boldsymbol{y}$ and $\mathcal{C}$. The physical implication of this is that the ground state energy $\lambda(\boldsymbol{y}, \mathcal{C})$ (per component) is lower bounded by the free energy $f(\beta ; \boldsymbol{y}, \mathcal{C})$ (per component) for an arbitrary temperature $\beta^{-1}>0$. In particular, the free energy $f(\beta ; \boldsymbol{y}, \mathcal{C})$ agrees with $\lambda(\boldsymbol{y}, \mathcal{C})$ in the zero temperature limit $\beta \rightarrow \infty$, which is the key for the analysis.

The distribution of the free energy $P(f ; \beta)$ is expected to peak at its typical value of

$$
f_{t}(\beta)=-\frac{1}{M \beta}\langle\ln Z(\beta ; \boldsymbol{y}, \mathcal{C})\rangle_{\boldsymbol{y}, \mathcal{C}}=\lim _{n \rightarrow 0} \frac{-1}{M \beta} \frac{\partial}{\partial n} \ln \left\langle Z^{n}(\beta ; \boldsymbol{y}, \mathcal{C})\right\rangle_{\boldsymbol{y}, \mathcal{C}},
$$


where $\langle\cdots\rangle_{\boldsymbol{y}, \mathcal{C}}$ denotes the average over $\boldsymbol{y}$ and $\mathcal{C}$, and decays exponentially away from $f_{t}(\beta)$ as $P(f ; \beta) \sim \exp [-M c(f, \beta)]$ for large $M$. Here, we assume that $c(f, \beta) \geq 0$ is a convex downward function that is minimised to 0 at $f=f_{t}(\beta)$. This formulation implies that, for $\forall n \in \mathbf{R}$, the logarithm of the moment of the partition function $Z(\beta ; \boldsymbol{y}, \mathcal{C}), g(n, \beta) \equiv$ $-M^{-1} \ln \left\langle Z^{n}(\beta ; \boldsymbol{y}, \mathcal{C})\right\rangle_{\boldsymbol{y}, \mathcal{C}}$, can be evaluated by the saddle point method as

$$
g(n, \beta)=\min _{f}\{n \beta f+c(f, \beta)\} .
$$

Based on the Legendre transformation ([6),$c(f, \beta)$ is assessed by the inverse transformation

$$
c(f, \beta)=\max _{n}\{-n \beta f+g(n, \beta)\},
$$

from $g(n, \beta)$, which can be evaluated using the RM analytically extending expressions obtained for $n \in \mathbf{N}$ to $n \in \mathbf{R}$.

The above argument indicates that the typical value of the distortion averaged over the generation with respect to $\boldsymbol{y}$ and $\mathcal{C}$ can be evaluated as

$$
\langle\lambda(\boldsymbol{y}, \mathcal{C})\rangle_{\boldsymbol{y}, \mathcal{C}}=\lim _{\beta \rightarrow \infty} \lim _{n \rightarrow 0} \frac{1}{\beta} \frac{\partial g(n, \beta)}{\partial n},
$$

and that the average error exponent $\alpha_{\{A, B\}}(D, R)$, which is an abbreviation denoting $\alpha_{A}(D, R)$ and $\alpha_{B}(D, R)$, can be assessed as

$$
\alpha_{\{A, B\}}(D, R)=\lim _{\beta \rightarrow \infty} c(f=D, \beta)=\lim _{\beta \rightarrow \infty}\left\{-n \frac{\partial g(n, \beta)}{\partial n}+g(n, \beta)\right\},
$$

where $n$ is a function of $\beta$ that is determined by the extremum condition of Eq. (77) as $\beta^{-1} \partial g(n, \beta) / \partial n=D$. Equations (8) and (9) constitute the basis of our approach.

When the above general framework was applied to the random code ensemble, which is not a practical coding scheme, but can exhibit optimal performance, the theoretical limitations the RDF and optimal error exponents derived in IT [4, 5] - were reproduced correctly [6, 7]. These results support the validity of our theoretical framework. In addition to consistency with the existing results, we demonstrated the wide applicability of our framework for the perceptron-based code in [7, 8], which indicated that the perceptron-based code can also saturate the theoretical limitations in most cases.

\section{ALGORITHM BASED ON BELIEF PROPAGATION}

Calculation using the RM implies that the perceptron-based code potentially provides optimal performance for binary memoryless sources. However, this is insufficient when it is 
necessary to obtain a compressed sequence $\boldsymbol{s}$ for a given finite length of original data $\boldsymbol{y}$.

For the perceptron-based code, the compression phase to follow the prescription (11) is computationally difficult because it requires a comparison over $O\left(2^{N}\right)$ patterns to extract the ground state for the relevant Boltzmann distribution $P_{\mathrm{B}}\left(\boldsymbol{s} \mid \boldsymbol{y},\left\{\boldsymbol{x}^{\mu}\right\} ; \beta\right)=$ $\exp \left[-\beta \mathcal{D}\left(\boldsymbol{y}, \tilde{\boldsymbol{y}}\left(\boldsymbol{s} ;\left\{\boldsymbol{x}^{\mu}\right\}\right)\right)\right] / Z\left(\beta ; \boldsymbol{y},\left\{\boldsymbol{x}^{\mu}\right\}\right)$. The Boltzmann factor is rewritten here for the sake of subsequent expressions as

$$
\exp \left[-\beta \mathcal{D}\left(\boldsymbol{y}, \tilde{\boldsymbol{y}}\left(\boldsymbol{s} ;\left\{\boldsymbol{x}^{\mu}\right\}\right)\right)\right]=\prod_{\mu=1}^{M} \Xi_{k, y^{\mu}}\left(\frac{1}{\sqrt{N}} \sum_{i=1}^{N} x_{i}^{\mu} s_{i}\right),
$$

where we define $\Xi_{k, y^{\mu}}(z)=\exp \left[-(\beta / 2)\left\{1-y^{\mu} \cdot f_{k}(z)\right\}\right]$. We require computationally tractable algorithms that generate a probable state $s$ from the Boltzmann distribution.

The BP is known as a promising approach for such tasks. It is an iterative algorithm that efficiently calculates the marginal posterior probabilities $P_{s_{l}}\left(s_{l} \mid \boldsymbol{y},\left\{\boldsymbol{x}^{\mu}\right\} ; \beta\right)=$ $\sum_{s_{i \neq l}} P_{\mathrm{B}}\left(\boldsymbol{s} \mid \boldsymbol{y},\left\{\boldsymbol{x}^{\mu}\right\} ; \beta\right)$ based on the property that the potential function is factored as shown in Eq. (10). In general, the fixed point of this algorithm generally provides the solution of the Bethe approximation [9] known in SM.

To introduce this algorithm to the current system, let us graphically describe this factorization, denoting the predetermined variables $\left(y^{\mu}\right.$ and $\left.\boldsymbol{x}^{\mu}\right)$ and compressed sequence $\left(s_{i}\right)$ by two kinds of nodes, then connecting them by an edge when they are included in a common factor, which can be expressed as a complete bipartite graph shown in FIG. 1.

On that graph, BP can be represented as an algorithm that passes messages between the two kinds of nodes through edges as

$$
\begin{aligned}
\hat{\rho}_{\mu l}^{t+1}\left(s_{l}\right) & =\sum_{s_{i \neq l}} \Xi_{k, y^{\mu}}\left(\frac{1}{\sqrt{N}} \sum_{i=1}^{N} x_{i}^{\mu} s_{i}\right) \prod_{j=1(j \neq l)}^{N} \rho_{\mu j}^{t}\left(s_{j}\right), \\
\rho_{\mu l}^{t}\left(s_{l}\right) & =\prod_{\nu=1(\nu \neq \mu)}^{M} \hat{\rho}_{\nu l}^{t}\left(s_{l}\right),
\end{aligned}
$$

where $t=1,2, \ldots$ is an index for counting the number of updates. The marginalised posterior at the $t$ th update is given as $P_{s_{l}}^{t}\left(s_{l} \mid \boldsymbol{y},\left\{\boldsymbol{x}^{\mu}\right\} ; \beta\right) \sim \prod_{\mu=1}^{M} \hat{\rho}_{\mu l}^{t}\left(s_{l}\right)$. Because $s_{l}$ is a binary variable, one can parameterize the above functions as distributions $\hat{\rho}_{\mu l}^{t}\left(s_{l}\right) \sim\left(1+\hat{m}_{\mu l}^{t} s_{l}\right) / 2, \rho_{\mu l}^{t}\left(s_{l}\right) \sim$ $\left(1+m_{\mu l}^{t} s_{l}\right) / 2$, and $P_{s_{l}}^{t}\left(s_{l} \mid \boldsymbol{y},\left\{\boldsymbol{x}^{\mu}\right\} ; \beta\right)=\left(1+m_{l}^{t} s_{l}\right) / 2$.

Since the computational cost for the summation in Eq. (11) grows exponentially in $N$, it is extremely difficult to perform this algorithm exactly. However, because $\boldsymbol{x}^{\mu}$ are 


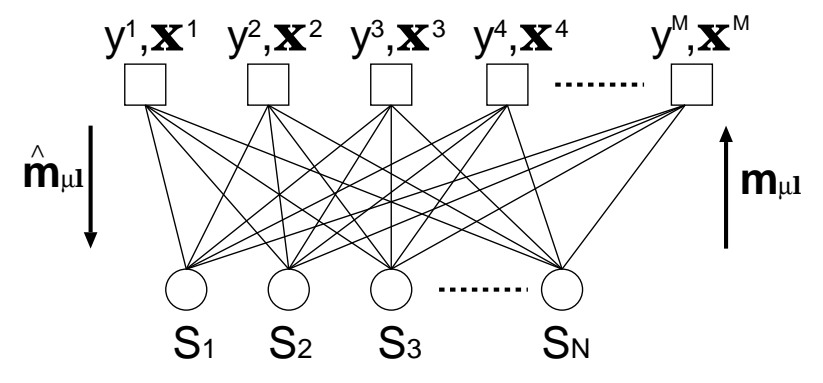

FIG. 1: Graphical representation of the dependency of variables for the perceptron-based code. Each pair of predetermined variables $\left(y^{\mu}, \boldsymbol{x}^{\mu}\right)$ is related with every bit of the compressed sequence.

generated independently from $P(\boldsymbol{x})=(\sqrt{2 \pi})^{-N} \exp \left[-|\boldsymbol{x}|^{2} / 2\right]$, this summation can be well approximated by a one-dimensional integral of a Gaussian distribution [10], the centre and the variance of which are $\Delta_{\mu l}^{t} \equiv\left(\sum_{i \neq l} x_{i}^{\mu} m_{\mu i}^{t}\right) / \sqrt{N}, 1-q_{\mu l}^{t}$, where $q_{\mu l}^{t} \equiv\left\{\sum_{i \neq l}\left(m_{\mu i}^{t}\right)^{2}\right\} / N$, respectively. This approximation makes it possible to carry out the belief updates (11) and (12) in a practical time scale, providing a set of self-consistent equations

$$
\begin{aligned}
\hat{m}_{\mu l}^{t+1} & =\frac{x_{l}^{\mu}}{\sqrt{N}} \frac{\int D z \Xi_{k, y^{\mu}}^{\prime}\left(\Delta_{\mu l}^{t}+\sqrt{1-q_{\mu l}^{t}} z\right)}{\int D z \Xi_{k, y^{\mu}}\left(\Delta_{\mu l}^{t}+\sqrt{1-q_{\mu l}^{t}} z\right)}, \\
m_{\mu l}^{t} & =\tanh \left(\sum_{\nu=1(\nu \neq \mu)}^{M} \tanh ^{-1} \hat{m}_{\nu l}^{t}\right),
\end{aligned}
$$

where we define $D z \equiv(d z / \sqrt{2 \pi}) \exp \left(-z^{2} / 2\right)$, and $f^{\prime}(x) \equiv d f(x) / d x$ for any function $f(x)$. Employing these variables, the approximated posterior average of $s_{i}$ at the th update can be computed as $m_{l}^{t}=\tanh \left(\sum_{\mu=1}^{M} \tanh ^{-1} \hat{m}_{\mu l}^{t}\right) \approx \tanh \left(\sum_{\mu=1}^{M} \hat{m}_{\mu l}^{t}\right)$.

The number of variables can be further reduced to $O(M)$ when $M, N$ is large by employing Eq. (14) [10]. One can approximately transform Eq. (14) into $m_{\mu l}^{t} \approx m_{l}^{t}-\left\{1-\left(m_{l}^{t}\right)^{2}\right\} \hat{m}_{\mu l}^{t}$. Utilising this equation and taking into consideration that the influence of each element in the sum is sufficiently small compared to the remains, the following approximations hold.

$$
\begin{aligned}
\Delta_{\mu l}^{t} & \approx \frac{1}{\sqrt{N}} \sum_{i=1}^{N} x_{i}^{\mu} m_{i}^{t}-\frac{1}{\sqrt{N}} \sum_{i=1}^{N} x_{i}^{\mu}\left\{1-\left(m_{i}^{t}\right)^{2}\right\} \hat{m}_{\mu i}^{t}-\frac{1}{\sqrt{N}} x_{l}^{\mu} m_{l}^{t}, \\
q_{\mu l}^{t} & \approx \frac{1}{N} \sum_{i=1}^{N}\left(m_{i}^{t}\right)^{2} \equiv q^{t} .
\end{aligned}
$$

Because the last term in Eq. (15) is infinitesimal, the Taylor expansion is applicable to Eq. 
(13), providing

$$
\begin{aligned}
\hat{m}_{\mu l}^{t+1} \approx & \frac{x_{l}^{\mu}}{\sqrt{N}} \frac{\int D z \Xi_{k, y^{\mu}}^{\prime}\left(T_{\mu}^{t}\right)}{\int D z \Xi_{k, y^{\mu}}\left(T_{\mu}^{t}\right)}-\left\{\frac{\int D z \Xi_{k, y^{\mu}}^{\prime \prime}\left(T_{\mu}^{t}\right)}{\int D z \Xi_{k, y^{\mu}}\left(T_{\mu}^{t}\right)}-\left(\frac{\int D z \Xi_{k, y^{\mu}}^{\prime}\left(T_{\mu}^{t}\right)}{\int D z \Xi_{k, y^{\mu}}\left(T_{\mu}^{t}\right)}\right)^{2}\right\} \frac{\left(x_{l}^{\mu}\right)^{2} m_{l}^{t}(17)}{N} \\
& \left(T_{\mu}^{t} \equiv \Delta_{\mu}^{t}-\frac{1}{\sqrt{N}} \sum_{i=1}^{N} x_{i}^{\mu} \hat{m}_{\mu i}^{t}\left\{1-\left(m_{i}^{t}\right)^{2}\right\}+\sqrt{1-q^{t}} z\right),
\end{aligned}
$$

where we define $\Delta_{\mu}^{t} \equiv\left(\sum_{i=1}^{N} x_{i}^{\mu} m_{i}^{t}\right) / \sqrt{N}$. It is important to notice that the second term of the right-hand side in Eq. (17), which can be negligible compared to the first term, becomes an influential element when the posterior average $m_{l}^{t}$ is calculated.

Using the new notations $a_{\mu}^{t}$ and $G^{t}$, the compression algorithm is finally expressed as

$$
\begin{aligned}
a_{\mu}^{t+1}= & \frac{\int D z \Xi_{k, y^{\mu}}^{\prime}\left(U_{\mu}^{t}\right)}{\int D z \Xi_{k, y^{\mu}}\left(U_{\mu}^{t}\right)}, \\
G^{t}= & \sum_{\mu=1}^{M}\left[\frac{\int D z \Xi_{k, y^{\mu}}^{\prime \prime}\left(U_{\mu}^{t}\right)}{\int D z \Xi_{k, y^{\mu}}\left(U_{\mu}^{t}\right)}-\left\{\frac{\int D z \Xi_{k, y^{\mu}}^{\prime}\left(U_{\mu}^{t}\right)}{\int D z \Xi_{k, y^{\mu}}\left(U_{\mu}^{t}\right)}\right\}^{2}\right], \\
& \left(U_{\mu}^{t} \equiv \Delta_{\mu}^{t}-\left(1-q^{t}\right) a_{\mu}^{t}+\sqrt{1-q^{t}} z\right) \\
m_{l}^{t}= & \tanh \left[\sum_{\mu=1}^{M} \frac{x_{l}^{\mu}}{\sqrt{N}} a_{\mu}^{t}-\frac{G^{t-1}}{N} m_{l}^{t-1}\right] .
\end{aligned}
$$

For the perceptron-based code, we can calculate

$$
\begin{aligned}
& \int D z \Xi_{k, y^{\mu}}\left(U_{\mu}^{t}\right)=e^{-\beta}+\left(1-e^{-\beta}\right)\left\{y^{\mu} H\left(w_{\mu-}^{t}\right)-y^{\mu} H\left(w_{\mu+}^{t}\right)-\left(y^{\mu}-1\right) / 2\right\} \\
& \int D z \Xi_{k, y^{\mu}}^{\prime}\left(U_{\mu}^{t}\right)=\frac{\left(1-e^{-\beta}\right) y^{\mu}}{\sqrt{2 \pi\left(1-q^{t}\right)}}\left[\exp \left\{-\frac{\left(w_{\mu-}^{t}\right)^{2}}{2}\right\}-\exp \left\{-\frac{\left(w_{\mu+}^{t}\right)^{2}}{2}\right\}\right] \\
& \int D z \Xi_{k, y^{\mu}}^{\prime \prime}\left(U_{\mu}^{t}\right)=\frac{\left(1-e^{-\beta}\right) y^{\mu}}{\sqrt{2 \pi}\left(1-q^{t}\right)}\left[w_{\mu-}^{t} \exp \left\{-\frac{\left(w_{\mu-}^{t}\right)^{2}}{2}\right\}-w_{\mu+}^{t} \exp \left\{-\frac{\left(w_{\mu+}^{t}\right)^{2}}{2}\right\}\right]
\end{aligned}
$$

where

$$
w_{\mu \pm}^{t}=\frac{ \pm k-\Delta_{\mu}^{t}+\left(1-q^{t}\right) a_{\mu}^{t}}{\sqrt{1-q^{t}}}, \quad H(x)=\int_{x}^{\infty} \frac{d z}{\sqrt{2 \pi}} \exp \left(-\frac{z^{2}}{2}\right) .
$$

The exact solution of $m_{l}^{t}(\forall l)$ trivially vanishes because of the mirror symmetry $\Xi_{k, y^{\mu}}\left(U_{\mu}^{t}\right)=\Xi_{k, y^{\mu}}\left(-U_{\mu}^{t}\right)$. This fact implies that one cannot determine the more probable sign of $s_{l}$ even if the update iteration is successful. A similar phenomenon was also reported in codes of another type [11, 12]. To resolve this problem, we heuristically introduce an inertia term that has been employed for lossy compression of an unbiased source 


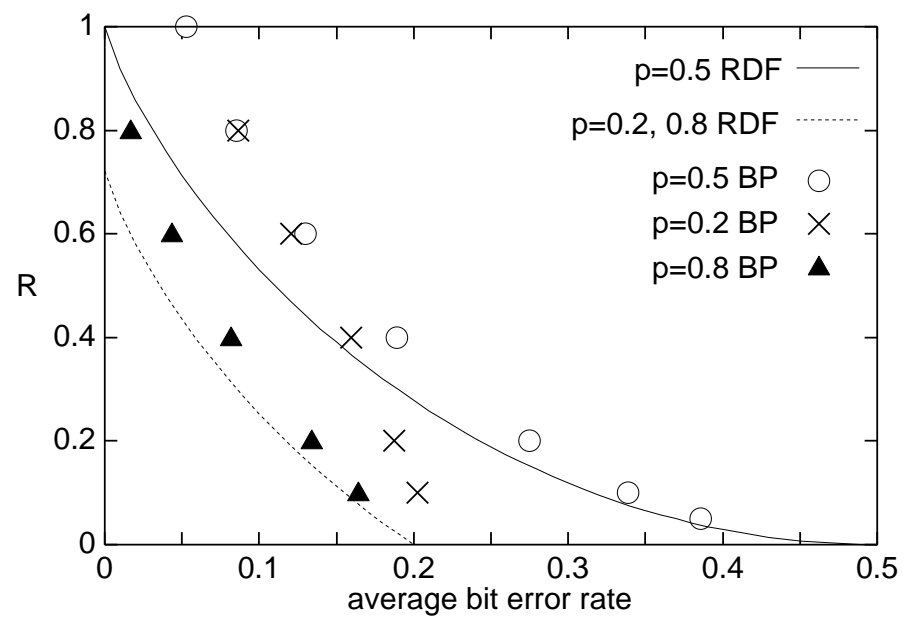

FIG. 2: Compression performance of BP for $p=0.2,0.5$ and 0.8. In the region of the low compression rate, the performance approaches the RDF in the case of $p=0.5$ and 0.8 .

11], in Eq. (201) as $m_{l}^{t}=\tanh \left[\sum_{\mu=1}^{M} x_{l}^{\mu} a_{\mu}^{t} / \sqrt{N}-G^{t-1} m_{l}^{t-1} / N+\tanh ^{-1}\left(\gamma m_{l}^{t-1}\right)\right]$, where $\gamma$ is a constant $(0 \leq \gamma \leq 1)$.

Experimental results are shown in FIG. 2 together with the RDFs. Given original data generated from a binary stationary distribution $P\left(y^{\mu}=+1\right)=1-P\left(y^{\mu}=-1\right)=p$ and vector $\left\{\boldsymbol{x}^{\mu}\right\}$, we compressed the original data into a shorter sequence using the BP. The final value of $s_{l}$ was determined as $s_{l}=\operatorname{sgn}\left[m_{l}^{t}\right]$. The values of $k$ and $\beta$ were set to theoretically optimal values evaluated from the RM-based analysis; the value of $\gamma$ was determined by trial and error. In the figure, the bit error rates averaged over 100 runs are plotted as a function of the compression rate $R$ for bias $p=0.2,0.5$ and 0.8 . While the compressed sequence was fixed to $N=1000$ bits $(N=500$ when $R \leq 0.2)$, the length of the original data was adjusted in accordance with the compression rate. We stopped the iteration at the 35th update and determined the compressed sequence from the result at that time, even if the algorithm did not converge. As the compression rate becomes smaller, the performance approaches the RDF in the case of $p=0.5$ and 0.8 . In particular, the performance for $p=0.5, R \leq 0.4$ is superior to results reported in the IT literature as a binary memoryless source [13]. However, the results for $p=0.2$ yield poor performance compared to those for $p=0.8$, even though the situation from the perspective of information is the same as $p=0.8$, which might be the result of asymmetric influences of input-output relations between those two cases. Improvement of this behaviour is a subject of future work. 


\section{SUMMARY}

We have investigated the performance of lossy data compression for uniformly biased binary data. Analyses based on the RM indicate the great potential of the perceptron-based code, which is also partially confirmed by a practically tractable algorithm based on BP. A close relationship between the macroscopic dynamics of BP and the replica analysis, was recently reported [10]. Investigation in such a direction in the current case is under way.

\section{Acknowledgments}

This research was partially supported by Grants-in-Aid Nos. 164453 (TH), 14084206 and 17340116 (YK) from JSPS/MEXT, Japan.

[1] C. E. Shannon, IRE National Convention Record, Part 4, 142 (1959).

[2] H. Nishimori, Statistical Physics of Spin Glasses and Information Processing - An Introduction, Oxford University Press, (Oxford), (2001).

[3] J. Pearl, Probabilistic Reasoning in Intelligent Systems: Networks of Plausible Inference, Morgan Kaufmann (San Francisco), (1988).

[4] K. Marton, IEEE Trans. Inf. Theory, 20, 197 (1974).

[5] I. Csiszár and J. Körner, Information Theory: Coding Theorems for Discrete Memoryless Systems, Academic Press Inc., p.158 (1981).

[6] Y. Kabashima and T. Hosaka, Prog. Theor. Phys. Suppl., No. 157, 197 (2005).

[7] T. Hosaka and Y. Kabashima, J. Phys. Soc. Japan, 74, 488 (2005).

[8] T. Hosaka, Y. Kabashima and H. Nishimori, Phys. Rev. E, 66, 066126 (2002).

[9] Y. Kabashima and D. Saad, Advanced Mean Field Methods, The MIT Press, p.51 (2001).

[10] Y. Kabashima, J. Phys. A: Math. Gen., 36, 11111 (2003).

[11] T. Murayama, Phys. Rev. E, 69, 035105 (2004).

[12] S. Ciliberti, M. Mézard and R. Zecchina, Phys. Rev. Lett., 95, 038701 (2005).

[13] E. Yang, Z. Zhang, and T. Berger, IEEE Trans. Inf. Theory, 43, 1465 (1997). 